Supporting information

\title{
Syntheses, Structures and Physical Properties of Three Novel Metal-Organic Frameworks Constructed by Aromatic Polycarboxylate Acids and Flexible
Imidazole-based Synthons
} Lili Wen, Zhenda Lu, Jianguo Lin, Zhengfang Tian, Huizhen Zhu, and Qingjin Meng*

Coordination Chemistry Institute, State Key Laboratory of Coordination Chemistry, Nanjing University, Nanjing 210093, P.R. China

E-mail: mengqj@nju.edu.cn 


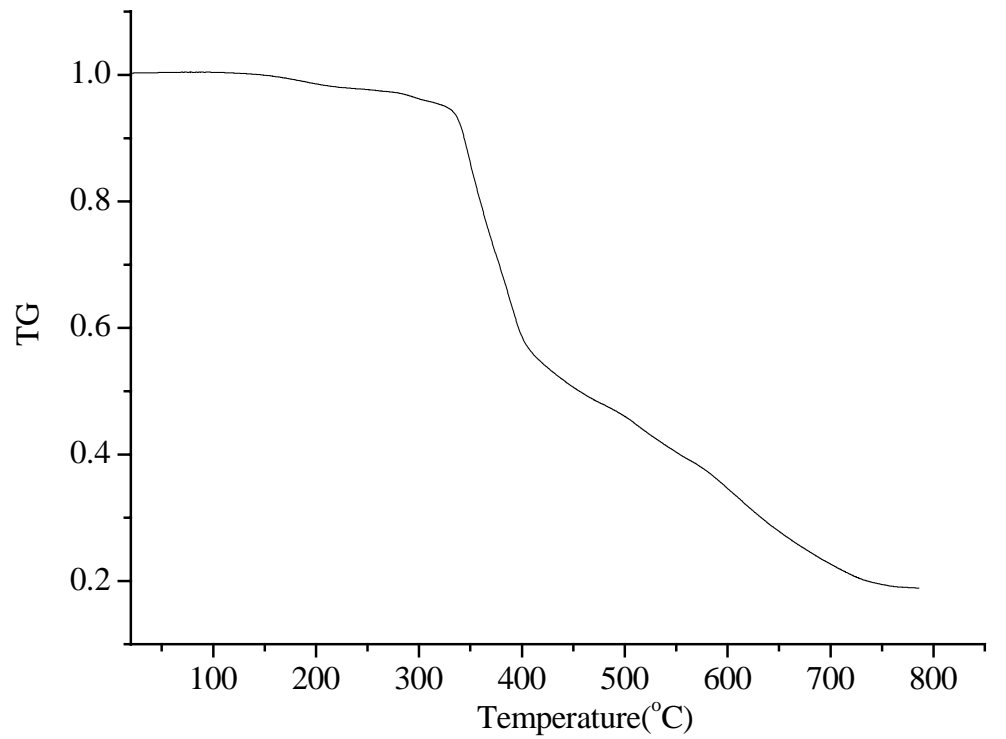

Figure S1. TGA curve of 1 .

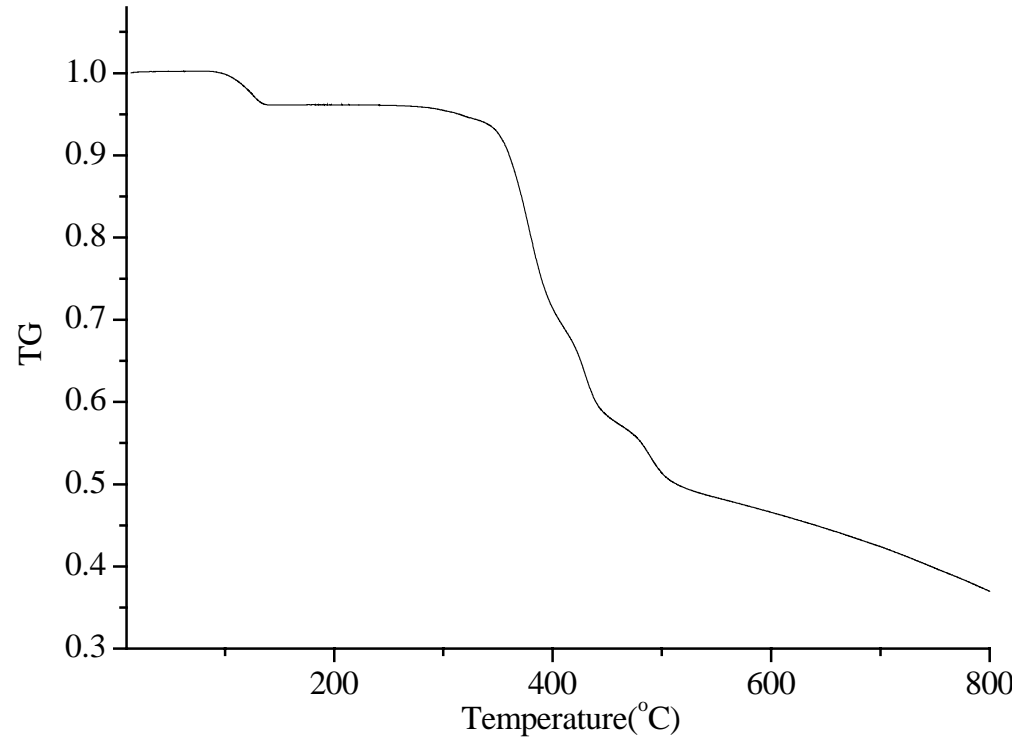

Figure S2. TGA curve of 2.

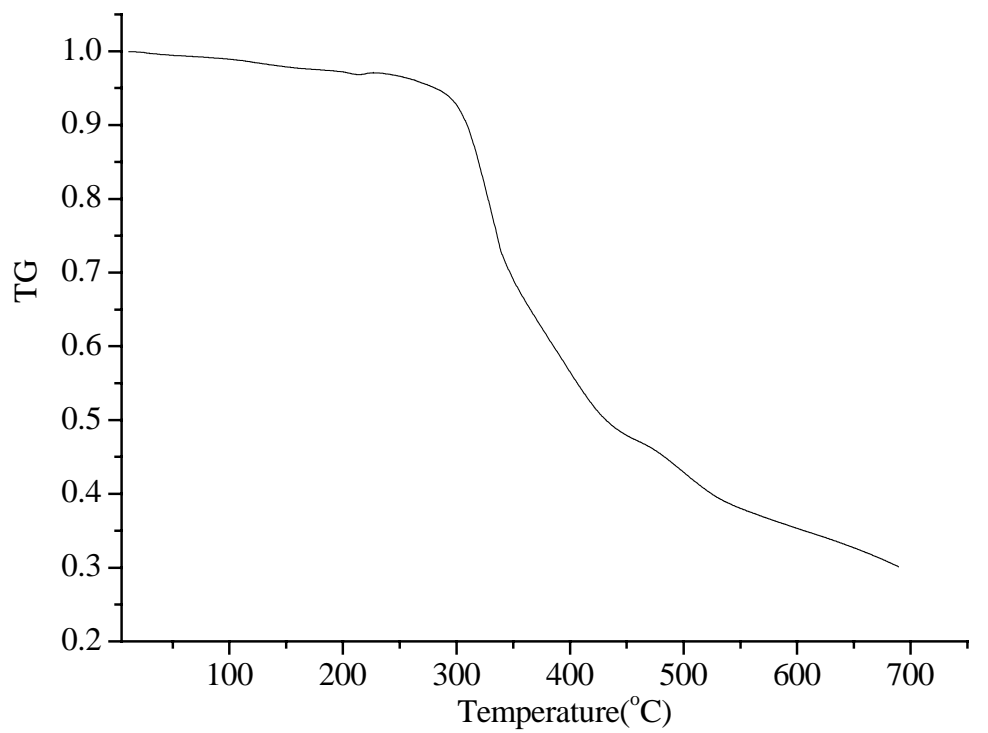

Figure S3. TGA curve of 3. 


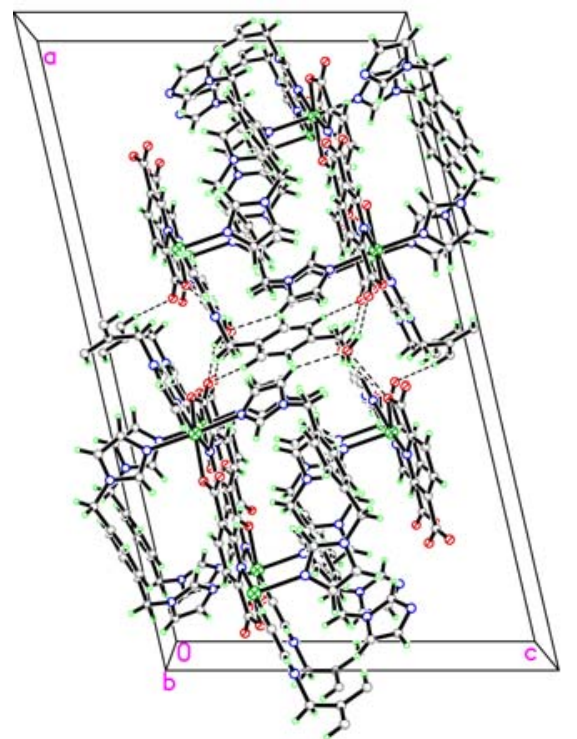

Figure S4. The packing diagram of a unit cell of $\mathbf{3}$ showing the hydrogen bonding interactions as dashed line. 